\title{
An ethnographic study of opioid use disorder in rural Maine: The problem of pain
}

\author{
Weronika Grabowska, ${ }^{1,3}$ Selma Holden, ${ }^{2}$ Peter M. Wayne, ${ }^{1}$ Karen Kilgore ${ }^{4}$ \\ ${ }^{1}$ Osher Center for Integrative Medicine, Harvard Medical School and Brigham and Women's Hospital, Boston, MA; ${ }^{2}$ University of \\ New England, College of Osteopathic Medicine, Biddeford, ME; ${ }^{3}$ College of the Atlantic, Bar Harbor, ME; ${ }^{4}$ School of Special Education, \\ School Psychology and Early Childhood Studies, University of Florida, FL, United States of America
}

\begin{abstract}
This qualitative study was conducted to more fully understand health care providers and community leaders' perceptions of the opioid crisis in rural Maine. In 2017, Maine continued to have one of the highest opioid overdose death rates in the country, more than double the national average. I (first author) visited eight treatment centers in Maine providing support and treatment to people recovering form Opioid Use Disorder (OUD), shadowing health care providers. I also attended OUD-related meetings held with community leaders. I conducted a total of 33 semi-structured interviews with health care providers, community leaders, and NGOs in the state of Maine. Three themes emerged integrating observations with semi-structured interviews: i) Impact of emergence of new extended release opioids, their prescription patterns, and culture around them; ii) Subjectivity of pain and importance of understanding psychic injury in OUD treatment; iii) Socio-political context and perception of OUD in Maine. Our society's perception of pain has deep historical and cultural sources that influence the way that pain has been perceived and treated in the medical setting. Resources beyond the medical environment are needed to address pain adequately.
\end{abstract}

Correspondence: Weronika Grabowska, Osher Center for Integrative Medicine, Harvard Medical School and Brigham and Women's Hospital, 900 Commonwealth Ave, Boston 02215, MA.

Tel.: 617-732-6271; Fax: 617-731-3843.

E-mail:wgrabowska@coa.edu

Key words: Opioid Use Disorder (OUD); rural health; ethnography.

Acknowledgements: Special acknowledgement to Netta van Villet, $\mathrm{PhD}$, the director of the undergraduate senior thesis research, on which the publication is based.

Contributions: WG: research design, data collection and analysis, main manuscript author; SH: research design, analysis consultation and manuscript editor; KK: manuscript co-author; PMW: manuscript co-author.

Conflict of interest: The authors declare no conflict of interest.

Funding: This project has been supported by Rothschild Fund for Thesis Research at the College of the Atlantic, and National Institute Grant K24AT009282 to PW.

Disclaimer: We recognize that use of stigmatizing language can further propagate stigma associated with substance use disorder, while the use of non-stigmatizing language is crucial to combat such stigma. Therefore, in this paper, we refrained from the use of words such as "addiction," "addicts" etc. whenever possible, replacing them with non-stigmatizing terminology.

All names used in the text are pseudonyms.

Received for publication: 12 June 2020.

Accepted for publication: 8 December 2020.

This work is licensed under a Creative Commons Attribution NonCommercial 4.0 License (CC BY-NC 4.0).

${ }^{\circ}$ Copyright: the Author(s), 2020

Licensee PAGEPress, Italy

Qualitative Research in Medicine \& Healthcare 2020; 4:132-145 doi:10.4081/qrmh.2020.9175

\section{Introduction}

"Problems? They'll take care of them on their own"

"On an early morning in February, I woke up to about two feet of snow, which had fallen during an overnight storm. I struggled to shovel my way out of the house and get a taxi to a meeting regarding a new opioid prescription law, Chapter 488 Legislation passed by the Maine Legislature during the $127^{\text {th }}$ session (2016), entitled, Prevent Opiate Abuse by Strengthening the Controlled Substances Prescription Monitoring Program. ${ }^{1}$ It was the first legislative effort in Maine to address the opioid crisis. During a break in the meeting about Chapter 488, I was reminded of an opioid use disorder story told to be me by Dr. Smith, a Primary Care Physician (PCP), about one of his clients.

Like many Down East fishermen, his client, Dominic, injured himself several times during his work. Never having time to fully heal, Dominic's injuries turned into chronic condition with severe back pain. Not able to afford time off, Dominic went to his PCP and received a prescription for one of the extended release opioids. His pain was relieved, and he was able to work again. The opioid medication was intended to be a temporary solution enabling Dominic to continue work. Yet when the summer season ended, the lobsterman was still using opioids, potentially even more than at the beginning. When the PCP discharged him from his medical practice, the lobsterman was already de- 
pendent and needed to find a substitute. Driven by horrific withdrawal symptoms, he initiated a search for other, often illicit opioids. Dominic entered the practice of this provider after a long journey involving the criminal justice system. I wondered why Dominic had not asked for medical help for his symptoms sooner. Later, Dr. Smith noted, that, "Mainers are proud people who don't like to ask for help. A lobsterman? That is probably the most 'Maine' you can get. They don't want to talk about their feelings." (Field notes, 2017)

Unfortunately, Dominic's situation is not unique. The problem of Opioid Use Disorder (OUD) in Maine is complex, embedded in a socio-historical context that defies quick or simple solutions. Maine, a predominantly rural state, relies heavily on seasonal manual labor in the fishing and lumber industries along with tourist-oriented services, crucial to its economy. Despite decent wages, many Maine residents are required to work several jobs during the summer to save money for the rest of the year. The intense summer season is followed by a long, dark winter, often accompanied by feeling of isolation caused by scarce social life in its small, scattered towns. According to Dr. Smith, Dominic did not have a steady income, and the misuse of opioid medication may have developed, in part, from a difficult social, economic, and geographic context. Other researchers exploring the phenomena of OUD in rural areas have also described socio-economic vulnerabilities that diminish the ability of people with OUD to seek treatment, particularly among those with low educational attainment, no health insurance, and low incomes. ${ }^{1-3}$ The purpose of this study was to explore the ecological perceptions of OUD among community leaders and health care providers in rural Maine.

\section{The larger context: The national opioid epidemic}

Opioid consumption has become a national crisis with opioid related deaths rising by 200 percent between 2000 and $2015 .{ }^{4}$ Since the early 2000s, the U.S. Public Health Services has noted an increase in use of opioid prescription medication (OPM). The Center for Disease Control (CDC) and the National Institute on Drug Abuse (NIDA) recognized the problem and urged implementation of policies restricting use of. ${ }^{5}$

Significant changes in opioid prescription and drug poisoning patterns took place in the United States between late 1980 and 2015, according to CDC data. ${ }^{6,7}$ In the 1980s, pharmaceutical companies developed new, refined formulations of Extended-Release (ER) opioids, thereby, changing "opioid-phobic" attitudes in the medical field at that time. ${ }^{8,9}$ Pharmaceutical companies provided evidence from multiple studies suggesting that medications such as OxyContin or Hydrocodone, used over an extended period of time, could effectively alleviate chronic pain and allow people to regain function. ${ }^{10}$ Furthermore, the studies indicated that ER opioids were unlikely to cause development of tolerance or dependance, allowing manufacturers to advertise them as less addictive., 3

Later, the pharmaceutical companies manufactured opioids with Abuse-Deterrent Formulations (ADF), intended to prevent people from crushing, snorting, and injecting ER opioids ${ }^{3}$ and thereby, to prevent abuse of the new generation of opioid medication. In practice, however, the intended value of these modifications proved to be ineffective as rates of OUD rose and patients suggested that their OUD often began with prescription medications, despite ADF technologies. ${ }^{11}$

\section{OUD in the rural state of Maine}

In recent years, Maine has had one of the highest national use of opioid dosage in morphine equivalent per capita $\left(798 \mathrm{mg} /\right.$ person). ${ }^{12}$ The state has also ranked among highest in use of ER OPMs in the country. ${ }^{12}$ In the early 2010 's, the increased prescription and use of opioids resulted in quickly growing numbers of opioid overdoses. Between 2011 and 2014, Maine observed a 34\% increase in the number of all drug related overdoses and a $340 \%$ increase in illicit drug-related overdose deaths. ${ }^{13}$ During the same period, the number of oxycodone related deaths averaged 41 per year, while heroin related deaths increased 5.4-fold and fentanyl related deaths increased 3.8fold. ${ }^{13}$ In Maine, as in many other states, males and females aged 25 to 54 were consistently the group with the highest proportion of overdoses. ${ }^{13}$

Although Maine reported a decline in both opioid prescriptions and prescription-related deaths, in 2017, Maine continued to have one of the highest opioid overdose death rates in the country (29.9 per 100,000); more than double the national average of 14.6 per 100,000. ${ }^{14}$ In 2017 alone, 278 out of 360 overdoses involved synthetic opioids, mainly fentanyl. ${ }^{14}$ Some evidence suggests that Maine's OUD crisis could be influenced, in part, by contextual factors specific to rural areas.

\section{Rural areas vulnerability to OUD}

Based on National Mortality Database, between 2006 and 2015, drug overdose mortality rates were higher in rural areas, including New England, compared to urban areas. ${ }^{15}$ In Maine, similar to other predominantly rural states, the OUD crisis has been exacerbated by a set of circumstances characteristic to remote, nonmetro areas.

Communities outside of metropolitan areas with low median household incomes and high unemployment rates have elevated opioid prescribing rates, suggesting that economic factors are important in fueling OUD. ${ }^{16}$ Over the last decade, rural areas experienced a decline in high paying jobs, which resulted in increased levels of hopelessness and lack of control among their inhabitants. ${ }^{17,18}$ Many rural areas have higher rates of manual labor, exposing workers to higher risk of physical injury and 
chronic pain. Historically, these regions have a longstanding acceptance of opioid use among workers who rely on pain medications to manage pain and continue working self-management. ${ }^{18}$

People struggling with OUD, residing in rural areas, often face problems regarding availability, acceptability, and accessibility of adequate OUD treatments. Rural areas often have a lesser number and density of specialized clinics and detox centers, offer less concurrent psychosocial services, and have limited numbers of providers. ${ }^{2,19,20}$ Moreover, rural areas struggle with providers' retention due to lower salaries, limited opportunity for continued education, longer hours, and few resources supporting their work, including funding for Medication-Assisted Treatment (MAT). ${ }^{17}$ Consequently, less than $10 \%$ of US providers with suboxone waivers practice in rural areas. ${ }^{19}$ Rural area providers are more likely to support abstinence only recovery, further limiting access to MAT. ${ }^{17}$ Joudrey et al. (2019) showed that the daily drive time to methadone clinic in rural areas increased on average by 30 minutes compared to micropolitan areas. ${ }^{20}$ Barriers of distance and time are further exacerbated by the scarcity of public transportation, a factor typical of Maine. ${ }^{2}$ Limited access, availability, and acceptability of treatment can lead to inconsistent and discontinued care, increasing chances for relapse. ${ }^{21}$

Rural contexts also impact social networks. People with OUD in rural areas tend to become members of social groups with fixed social identities. These distinct micro-social environments enable copying and spreading of drug seeking behaviors and quicker diffusion of illicit prescription opioids among the members, ${ }^{18,22}$ making them more vulnerable to OUD. Tightly interconnected social environments also heighten users' perceptions of stigma, preventing groups of users from seeking help during overdose or participating in harm reduction programs. ${ }^{22}$ Moreover, stigma present in the community can lead to a significant discrimination and exclusion of people labeled as "addicts," affecting their social status, employment prospects, criminal justice encounters, and access to health care..$^{22}$ Rural social networks introduce a paradox, where, on one hand, strong social connectedness can provide support needed in recovery, while on the other, it can increase the spread of negative behaviors and heighten stigma and exclusion.

\section{Purpose and research questions}

The aim of this study was to understand the perspectives of local community leaders and health care providers regarding OUD in rural Maine. In their everyday work, health care providers and community leaders negotiate treatment recommendations, policy, funding to provide their patients with the best standard of care. Through inperson interviews, the following open-ended, guiding questions were posed to direct data collection and analysis: i) What are the perceptions of community leaders and health care professionals regarding the development and progression of OUD in Maine? ii) What are the perceptions of community leaders and health care professionals regarding strategies for responding to the opioid problem?

Additional probing questions included: interviewees' professional and personal experiences with the opioid epidemic; their views on the current state of treatment and other resources offered to people with OUD, their availability and effectiveness; common stories of OUD development; identification of the biggest challenges OUD posed to individuals and the larger Maine community; their views on the historical and current approaches to combat OUD (Appendix).

\section{Materials and Methods}

To discern the perceptions of community leaders and health care providers, I (first author) conducted an ethnographic field study, collecting field notes, facilitating qualitative, semi-structured interviews, and informal interviews in the field. Ethnographies, rooted in anthropological methods, were designed to capture a holistic picture of how participants structure and interpret their lived experiences. The study was designed to capture participants' understandings of the complexity of the problem and uncover the health care challenges related to OUD treatment through in-depth understanding of the problem. ${ }^{23,24}$

\section{Data collection}

\section{Participant observations}

During this study, I visited eight different treatment centers providing support and treatment to people recovering from OUD to shadow medical practitioners or observe group therapy, to better understand the OUD problem in its socio-cultural context and too learn about types of OUD treatments and their accessibility. Prior to conducting observations, I contacted each site and asked the practicing providers for permission to observe. Research objectives were revealed to the clients and their consents were sought prior to conducting observations.

\section{Field notes}

Field notes were used to document observations and record my reflections on events during participant observations, including the flow of the sessions and conversations. I did not address the clients directly nor interfere with the providers, unless either of them addressed me directly. During some of the individual sessions, clients were encouraged by the providers to share their stories of OUD. To protect the privacy of the clients, these stories are not shared in any publicly available materials. These accounts, however, informed the process of data analysis. The methods employed in participant observation allow the researcher to ask questions as they emerge in the natural context under study. The researcher becomes the instrument, responding unobtrusively, asking participants 
about their lived experiences and noting interactions among participants in their naturally occurring setting. ${ }^{23,24}$ The responses to these informal questions and observed interaction were recorded in field notes.

Each shadowing session was conducted in full HIPAA compliance and in adherence to privacy requirements of each individual clinic and recovery center. No identifiable information was recorded or shared. The names of majority of interviewees and all their recalled clients were replaced by fictional names. All notes and transcripts were de-identified to protect participants' anonymity.

This research has been approved by the Institutional Review Board at the College of the Atlantic.

\section{Semi-structured interviews}

Semi-structured interviews were conducted with representatives of medical professionals, counselors, therapists, community leaders, law enforcement, and NGOs in the state of Maine, using purposive sampling to identify and recruit interviewees with significant experience related to substance use. Generalizability is not an aim of qualitative research, and does not require a sample representative of a larger population. Purposeful sampling is intended to identify and include individuals knowledgeable about the research topic. These individuals may suggest other respondents, who are informed or influential regarding OUD in rural Maine. ${ }^{23,24}$

Out of 57 contacted people, 33 representatives were interviewed in 30 individual sessions and one focus group. On average, the interviews lasted for one hour and 15 minutes. During participant observation, informal interviews were held with clients who approached me as I collected field notes, to better understand their attitudes towards Maine's treatment facilities. Consistent with purposive sampling, some respondents suggested interviews with other stakeholders, who were knowledgeable regarding OUD in rural Maine. As data collection and analysis progressed, in an iterative and reflexive process, themes regarding the interplay among medical professionals and treatment providers became stronger and more compelling. Ongoing data collection focused on exploring these themes. As noted above, the first author as a participant observer, was able to ask questions unobtrusively while in the field. We continued to collect data from our informants until data saturation was achieved. ${ }^{23,24}$

\section{Artifacts}

Copies of Maine legislation as it pertained to opioid use, pamphlets used in treatment centers to inform patients and their relatives about available treatment and support options, educational materials related to opioid prescriptions given out in clinics to patients, and articles from local newspaper featuring stories of OUD, overdoses, and their repercussions for Maine communities were also collected and their content helped informed the context of the research.

\section{Data analysis}

Thematic analysis, employing a constant, comparative method as described in grounded theory, was used to analyze the data. ${ }^{25,26}$ First, induction was used to develop ten narrative vignettes to determine the emerging themes. ${ }^{27}$ Then the transcripts and observational data were crossexamined, coded, and arranged into emergent themes. We continued to collect data as themes emerged, as further questions developed in our efforts to delve more deeply into the phenomena under study. The developed themes were discussed with the full research team (all authors) and related to the post-hoc literature review on opioid epidemic in the rural US, available epidemiology data form Maine, as well as OUD development and treatment theories. Data collection and analysis were guided by the Criteria for Reporting Qualitative Research (COREQ) proposed by Tong, Sainsbury, and Craig (2007). ${ }^{28}$

\section{Results}

The perceptions regarding the emergence of OUD in Maine shared by health care providers and community leaders are presented and summarized in Table 1. These findings present a complex array of factors contributing to the opioid epidemic in Maine.

\section{The dilemmas posed by ER opioids, managing chronic pain, and treating OUD}

\section{The dilemma of the new class of ER opioids}

As I volunteered and shadowed at one of the rural, critical access hospitals on the coast of Maine, I witnessed patients admitted with drug overdose or in withdrawal. I became privy to patients' stories about the development of their Substance Use Disorder (SUD) and how it challenged their everyday lives. I shadowed Dr. Carter, a PCP with a suboxone waiver. When asked about the opioid epidemic, Dr. Carter indicated that the manufacturing of ER opioids and their advertisement as "safe" were directly linked to over-prescription patterns among physicians. Dr. Carter argued that the rapid "incorporation of opioid prescriptions" into everyday medical practice was one of the major causes leading to the vast availability and accessibility of opioids. Dr. Carter recalled how the new generation of opioids was introduced:

"In retrospect, it turned out it was the pharmaceutical industry that pushed for such shift to make profits from their long-term acting opioids such as OxyContin. That was the case in the 90s [...] and everywhere you looked that's what you were hearing. So, unfortunately, doctors believed it and incorporated it into their practice. The opioid crisis really got started thanks to the pharmaceutical companies." (Carter, 2017). 
I also shadowed the medical practice of a psychiatrist at a family mental health clinic (The Clinic) in Down East Maine, who specialized in SUD recovery. The executive director of the Clinic, Dr. Davis, explained his view of OUD due to over-prescription, saying, "Due to their properties, use of opioids leads to development of tolerance, defined as a diminished response to the same drug dose." Tolerance, he said, is followed by drug dependence and addiction, both physically and psychologically. Consequently, the patient will need higher dosages to achieve the same effect. Tolerance develops over different periods of time in different patients. According to Dr. Davis, the over-prescription of OPM was a common story leading to SUD. As highlighted by the interviewed providers, opioid over-prescription became part of medical practice due to persistent pharmaceutical advertisement coupled with changes in the medical community regarding the significance of pain.

\section{The dilemma of pain as the "fifth vital sign"}

Physicians described another factor influencing overprescription of pain medications. In the 1990s, the American Medical Association recognized pain as the fifth vital sign. In terms of diagnosis, pain received equal status with the four other vital signs: blood pressure, heart rate, respiratory rate, and temperature. The implementation of the pain scale, the impetus to provide adequate pain treatment, and the new generation of ER opioids, coalesced into over-prescription of opioids. Dr. Carter. explained,

\begin{abstract}
"This epidemic was created by prescribing physicians. This is not something that grew up out of inner cities or mafia syndicates. This was entirely created by doctors and pharmaceutical companies... And here we are, 20 years later dealing with the fallout from this kind of corporate, greed-based decisions, which are taking advantage of doctors being empathetic and gullible and focused on treating their patients' pain... It is a very sad chapter in the history of modern medicine." (Carter, 2017).
\end{abstract}

Dr. Smith and Dr. Miller, physicians practicing in rural clinics, added that many of their Maine colleagues did not recognize the seriousness of the opioid over-prescription problem as it developed. Both MDs were dismayed by the continued absences of their colleagues (other medical professionals, whose patients were affected by OUD), in local meetings regarding the opioid epidemic and new legislation, Maine Chapter 488, designed to address the opioid crisis in Maine.

\section{The dilemma of OUD as a chronic disease}

The Clinic in Down East Maine, specializing in substance use recovery, offered an outpatient based on weekly or bi-weekly individual counseling sessions in combination with MAT. MAT utilizes partial or full opioid receptor agonists, such as methadone or buprenorphine; these pharmaceuticals help stabilize patients in recovery, help manage their cravings, and lower the rates of relapses. ${ }^{29}$ Dr. Davis, who treated Dominic, the lobsterman described in the introduction, worked alongside other providers at the Clinic, to stabilize their patients in recovery though MAT and individual counseling. Dr. Davis was one of the few MDs who had a suboxone waiver, allowing him to prescribe the opioid replacement medication to his patients in recovery. Dr Davis believed that suboxone enabled his patients to quit "chasing the drugs," providing them with an opportunity to regain control over their lives, secure a job, and reestablish relationships with their family and friends. Dr. Davis believed, that ideally, patients in recovery should graduate from MAT and not return to opioids.

Whether MAT alone was able to prevent one from relapsing, however, was unclear to treatment providers, as some of them expressed concerns that the medical model of treating opioids had limited usefulness. Yet, Dr. Smith, described the importance of comparing OUD to a chronic disease, such as diabetes. He explained,

\begin{abstract}
"Diabetes is not the best analogy to use, but the analogy offers an opportunity for PCPs [to describe opioid] addiction as a chronic disease which requires long-term treatment. And just as diabetics often relapse and do not strictly follow their diet, addicts in recovery also slip and are not always successful right away. It also aids with creating treatment options. No one would withhold insulin from a diabetic, right? [...]. I think, it also helps decrease the stigma around it." (Smith, 2017).
\end{abstract}

Several of Dr. Smith's colleagues also used the diabetes analogy to describe needed OUD treatment. Simultaneously, they criticized and disliked any legislative efforts that could intrude into the doctor - patient relationship limiting treatment options, such as the Maine Public Law Chapter 488. ${ }^{1}$ Nevertheless, Dr. Smith, however, believed that addressing OUD solely as a diabetes-like chronic disease did not adequately capture the full picture of the disorder.

\section{Socio-political context of OUD in Maine}

Maine Public Law Chapter 488: An act to prevent opiate abuse

Maine Public Law Chapter 488 (2016) introduced a prescription monitoring program and limited physicians' treatment options for prescribing opiates. ${ }^{1}$ Several health care providers, as noted above, expressed frustration with aspects of the public law. Dr. Jones explained that the law limited options for his "relapsing" patients.

"I think that whenever the government gets involved in [medical] treatment, it is inappropriate. They do not tell me if a diabetic patient doesn't 
take their medication appropriately and relapses, that I can't treat them. They don't tell me that the person with hypertension, who smokes and does not exercise, who then has a stroke, has a limit on how long we take care of them. Why offer these arbitrary rules? It is driven by dollars and not by scientific data. We can take the attitude that we need to balance the budget. But even then, if you don't treat addicts, the cost to the society and the cost of the incarceration can be 10 times higher than the cost of treatment." (Jones, 2017).

Dr. Jones described the difficulties of interweaving of medical practice with policy as it relates to OUD. The Maine legislators, who developed Chapter 488, introduced a possibility for prescribers to award patients in recovery with a "relapse" status, prolonging their possibility to remain in treatment for another 6 months. Nevertheless, study informants said that the time limitations hampered physicians' abilities to intervene appropriately with more adequate resources.

Since the time of conducting the interviews, Chapter 488 has been modified. Under section 20.32, MAT was classified as an exception, allowing physicians to continue treating their patients with OUD without arbitrary limitations on the length of MAT prescriptions. ${ }^{1}$

\section{The criminalization of $O U D$ and the introduction of heroin}

Patients who relapsed after short-term treatment or lack of treatment sought opioids elsewhere and often ended up in the criminal justice system. Dr. Carter observed:

"In the 90s there was an explosion of prescription opioids, and a lot of poor rural people got addicted that way. When people got addicted to prescription medication, prescription opioids became more expensive and less accessible, and at that point, heroin diffused from inner cities and became more accessible in the rural areas [...]. I think that heroin addiction is a new phenomenon in the rural areas, and I think it stems out of the opioid prescription use, because I don't think there was any other wave of heroin "pushers" who would move from the urban areas to come and introduce heroin here. Heroin filled the void, when prescription medication became less available. These things come in waves. So now we have fentanyl on the rise." (Carter, 2017).

\section{Dr. Jones offered a similar view:}

"Another thing we need to think about is why the heroin street price went down. What caused that? What made it so readily available? So, you start with a bunch of OxyContin from the doctor, you develop the disease of addiction - now you are dependent. Then you want to continue either with opioid medications or heroin. And so, at first, heroin was very expensive, and it was hard to get. There was not much supply available on the market. [...] But as the prescription medications started to be more expensive and harder to get, heroin was becoming otherwise." (Jones, 2017)

Heroin, however, was the drug of "last resort" for many people with OUD. Heroin posed one major challenge: to be used, it had to be injected, which was a much less appealing way of administration than simply taking pills. Dr. Jones believed that heroin providers found a solution to make it more "user-friendly."

"People who sell heroin are not stupid. They know that clean heroin can be snorted and that could be appealing for people who have [an] aversion towards needles. So, all in all, the continuation of the epidemic we are observing right now is possible, because people were able to switch to cheaper, better [more potent], and more available heroin." (Jones, 2017).

Statistics supported these physicians' views. Heroin increasingly became the cause of overdoses and deaths throughout Maine. ${ }^{11,13}$ With the introduction of heroin, the opioid epidemic, which presumptively began in a medical setting, moved onto the streets and became highly stigmatized and criminalized. The change in venue led to involvement of law enforcement and further criminal legislation, impinging upon the doctor-patient relationship.

Dr. Davis was deeply disturbed to see his patients become destabilized and relapse due to short stays in jail. Moreover, he did not have access to his clients while incarcerated. Consequently, patients whom he had treated with suboxone were inaccessible to him and unable to receive suboxone. He wondered, "Why can't [law enforcement agencies] give them the suboxone? It's a medication after all. They would not take away an inmate's insulin." According to Dr. Davis, the criminalization of clients with OUD also resulted in further stigmatization, prompting exclusionary processes that marginalized people under his care. Dr. Smith noted that the difficulty of treating OUD patients stemmed from historical prejudices and social stigma associated with SUD. The strict engagement of law enforcement created a conflict between their representatives and the medical field.

During an informal meeting at the Clinic, health care providers and counselors noted another persistent concern regarding their clients, many of whom seemed overwhelmed with everyday tasks, such as paying bills, controlling finances, managing a household, or being consistent with their children. Due to limited resources and time constrains, the counselors concluded that the 
Clinic could not address these basic life skills and that some of their clients may need social workers for additional support. However, they also noted that those services were extremely difficult to obtain in Maine. It became clear that the problem of OUD was more complex than addressing chronic pain, physiological addiction, and offering MAT.

\section{The problem of pain}

\section{OUD arising from psychological as well as physical injury}

Health care providers and counselors described a multitude of factors fueling the OUD in Maine. These included a lack of healthcare insurance, an absence of adequate social support (public transportation, childcare), an absence of family support, a lack of sustainable employment, and isolation. It became clear that providing people with MAT, though vital in the initial phase of recovery, would not solve the problem alone. In most cases, chronic pain could not be solved solely through medical means. Dr. Davis explained,

"Chronic pain is a really tough problem and opioids are not the solution. Chronic pain is a difficult enough problem to manage by itself. Other socio-economic factors, psychiatric issues, and societal problems add to it, making it even more difficult to manage... There are people who have legitimate chronic pain problems, and I do have a few patients in my practice, who are reasonably well managed on a reasonable dose of chronic opioid that have not run into any major problems with their opioid therapy. Unfortunately, the downside is that, I think, most of the patients are not well managed and [opioid therapy] is not a very good treatment for [their] chronic pain." (Davis, 2017).

Given that the over-prescription of medications began as an attempt to address chronic pain, Dr. Davis directed his attention towards the nature of pain. According to him, chronic pain is much more complicated than most physicians understand.

\footnotetext{
"Opioids do not only treat physical pain. They treat emotional pain. In a sense, you want to broaden the definition of injury to psychic injury, or emotional injury, like trauma, depression, profound anxiety. Those are emotionally painful situations, and I don't think those get fully appreciated. And so, what the [patients] are looking for is an escape [from different kinds of pain] and opioids provide that. In a very, very short term it is a solution, as their pain diminishes. Then, very quickly they become addicted [...]." (Davis, 2017).
}

Mental health providers recalled stories of trauma and emotional pain experienced by their patients with OUD.

For example, Dr. Smith suggested that defining substance use as a predominantly medical problem can help to de-stigmatize OUD and advocate for better access to its medical treatment. However, in further conversations, he noted that medical interventions alone cannot address the structural changes required to provide more supportive environments, alter social attitudes regarding OUD, and remove barriers to wider prevention and treatment.

Dr. Williams, the director of the outpatient methadone clinic in one of the psychiatric hospitals in Maine, commented directly on the role of both physical and psychic pain in OUD. Dr. Williams placed emotional trauma in the context of self-medication and SUD.

"I hear people say, "I was using to feel numb. Now I'm not using and life hurts." And it's very common for people with trauma to say that, and it makes it much more difficult for them to engage in recovery, cause it still hurts. And they don't know how to deal with their pain. The only thing they've learned over the years is that "this pill [opioids] takes care of my emotional pain. But when I don't have this pill, how am I going to take care of my emotional pain?" They will need to take time to learn how to take care of their emotional pain. And during that time, it's always a struggle." (Williams, 2017).

Unresolved, underlying emotional distress triggers relapsing. ${ }^{30}$ Healing requires time during which people may present with variable, interchangeable, psychological and physiological symptoms. When in treatment for OUD, the opioid-induced numbness disappears, and the feelings associated with trauma return. In the absence of support for addressing underlying trauma, relapses in treatment become more frequent.

When asked about the common stories of SUD, interviewees would often talk about underlying trauma, domestic violence, physical and sexual abuse. Though, emotional pain seems to accompany OUD often, the stories about prevailing pain and the difficulty to move on are frequently not included in statistics or biomedical studies. The stories of emotional pain came up repeatedly during the interviews with healthcare providers and community leaders, who would describe the devastating repercussions such events had on people's lives. During participant observation in recovery centers across the state, patients would frequently mention past abuse, neglect, and trauma as events leading up to or reinforcing their OUD. The prevalence of these stories led to further questions for health care providers about their perspective on emotional pain in the context of the current opioid epidemic. One mental health counselor explained,

“Trauma is huge. It's either because a client grew 
up in a home, where because of the use and abuse, they lived in the environment of violence and all of that that comes with it; or they ended up living with individuals with high risk, and so they might have experienced multiple rapes, assaults, or domestic violence. Sometimes even being on the street where you don't know when your next meal will be and what is going to happen, or simply witnessing things that are violent can be very traumatic. Many of our clients come from homes where there have been emotional, psychological, and physical abuse. [...] And every woman that comes to our residential Hills program has severe trauma; every one of them." (Rose, 2017).

Trauma leads to susceptibility to SUD, and SUD fuels further trauma. To illustrate the prevalence of trauma, Dr. Williams, linked trauma to the degree of severity of one's OUD.

"So [about trauma], it is something that I hear about, time and time again, especially with the methadone and suboxone patients. We tend to get patients with the most severe addiction. People with milder addiction would go to their PCP or they would stop [taking drugs] themselves. They don't need replacement therapy. With severely addicted people, none of these works. [...] What we see in people with very severe addictions is a common thread of trauma." (Williams, 2017).

Dr. Williams continued describing trauma in terms of emotional pain and the challenges it posed to many of his patients. Given how ubiquitous trauma was among patients who develop OUD,

Dr. Williams described the relationship between the two as causal:

"Looking at one's [medical] history, we can almost say that you can start perceiving trauma as predictive for severe addiction. Most people with trauma tell us: "when I took the pill for the first time, I felt different. I felt alive, I felt normal. For the first time I was living without pain." (Williams, 2017).

Dr. Williams further explained how trauma leads to the development of OUD.

"Opioids treat emotional pain as effectively as they treat physical pain. Pain itself has a strong emotional component. There is no such difference as physical and emotional pain, mind versus body. I don't think that there is much difference between mind and body, it's still the same person and it is the same pain. So [opioids] treat emotional pain. And trauma puts people more at risk for opioid ad- diction as trauma causes that emotional pain in the first place [...]." (Williams, 2017).

Dr. Williams did not distinguish between emotional and physical pain, but rather, portrayed trauma as making an individual more vulnerable to the potent analgesic effect offered by opioids. Dr. Williams raised the issue of mind-body dualism in Western medicine, the idea that the mind and body are two distinct entities with each having a different, essential nature. ${ }^{31}$ Dr. Williams suggested that pain should be considered and treated more holistically. Other components to pain, whether chronic or acute, need to be taken into consideration to explore the sources and design the solutions to end the opioid epidemic.

Dr. Smith expressed similar views to Dr. Williams. In considering the treatment of chronic pain, Dr. Smith stated,

"Chronic pain has biological, psychological and physical components in it. And if we're only dealing with the acute medical issue and not dealing with the psycho-social aspect of the problem, we will never resolve it fully, because there are so many driving forces to use or abuse temporarily or chronically opioids." (Smith, 2017).

Other respondents, in describing the complexity of chronic pain, also raised concerns regarding the role of social rejection or exclusion in exacerbating OUD through the social component of pain.

\section{Vulnerability to OUD arising from influence of social isolation, exclusion, and stigma}

The Peer Recovery Center in Portland operated differently from other recovery centers described previously. It is not associated with a formal treatment center and does not offer MAT to people in recovery. The center's focus is providing social support, understanding, and acceptance. Mr. Corteau, the director, and his colleagues interacted with clients as peers, emphasizing the importance of community support in recovery; they regard the medical model of SUD as secondary. Peer recovery, at the Peer Recovery Center, was about the people "who have been there," people who can relate and provide a different level of trust and understanding in comparison to the dynamics in other medical or counselling milieus. Corteau explained, "the opposition to addiction is not sobriety, but social connection." Combining the perspectives of a provider and person in long-term recovery, Corteau explained the role of social stigma in terms of personal shame and guilt.

"Shame is huge in addiction. While in active addiction, we use it in a maladaptive way to keep the addiction cycle going. So, I might do something in my active addiction that causes shame. For in- 
stance, I stole something, or I abused someone in some way and then I felt bad about it. The way that we cope with any emotion when we are in active addiction is we use more substances. So, in a maladaptive way shame is useful. It is part of the cycle of using. Sometimes, when people are trying to help, they can cause a little bit of that shame, often not purposefully. But when you do that, especially to those who struggle, you actually help fuel the addiction. So, the more we can combat that feeling of shame, the more we can propel person into recovery. Stigma is part of shame. If I stigmatize you, I am shaming you, right?” (Cotreau, 2017)

Relationships between people struggling with OUD and members of their communities are often damaged.

Stigma associated with substance use occurs across settings. For example, medical communities tended to stigmatize persons with OUD. Patients dependent on prescription medications who appeared in emergency departments or in primary care were often referred to as "frequent flyers", and "drug seekers," and were not necessarily treated compassionately or referred to treatment resources. Stigma, triggering shame and social isolation, contributed to cycles of relapse and OUD.

Mrs. Moore, a counselor who worked with inmates in the Hancock county jail, also described the conflicted relationships between those struggling with OUD and members of their rural communities, saying:
"There is hurtful behavior going around because of the addiction, as people are not in touch with their emotions. They are numbing out through using, so they are not in touch with many sides of themselves, which only brings them further away from their community and family. It is very alienating and builds walls around them; violates their dignity. [...] It is hard to see how addiction is a reaction to life challenges for those who do not have other toolkits to solve them. Our culture does not provide people with those skills." (Moore, 2017).

Moore addressed how the social context and self-detachment of the person with OUD creates a vicious cycle of hurt. A person suffering with OUD needs organized community support. Yet after years of hurtful behavior, broken trust, followed by exclusion, self-isolation, and experiences of shame, it can be difficult to find the needed support in already small and isolated Maine communities. Moore and Corteau comments raise the question: How can the cycle of OUD be broken through reconnecting people with each other and with themselves?

\section{Discussion}

\section{Similarities between rural Maine and the nation}

Several findings from this study (see Table 1) are similar to other published reports regarding OUD. As de-

Table 1. Themes and supporting quotes.

\begin{tabular}{|c|c|c|}
\hline Theme & Domain & Supporting quote \\
\hline \multirow[t]{3}{*}{$\begin{array}{l}\text { The dilemmas posed by extended-release opioids, } \\
\text { managing chronic pain, and treating OUD }\end{array}$} & The dilemma of the new class of ER opioid & $\begin{array}{l}\text { "Pharmaceutical companies pushed for the use of } \\
\text { ER opioids, and doctors, who believed in their } \\
\text { safety, incorporated them into practice." }\end{array}$ \\
\hline & The dilemma of pain as the "fifth vital sign" & $\begin{array}{l}\text { "[This is] a fallout from [...] corporate, } \\
\text { greed-based decisions, which are taking } \\
\text { advantage of doctors being empathetic and } \\
\text { gullible and focused on treating }[. . .] \text { pain." }\end{array}$ \\
\hline & The dilemma of OUD as a chronic disease & $\begin{array}{l}\text { "Addiction as a chronic disease requires a } \\
\text { long-term treatment, }[\ldots] \text { addicts in recovery slip } \\
\text { and are not always successful right away." }\end{array}$ \\
\hline \multirow[t]{2}{*}{ The socio-political context of OUD in Maine } & $\begin{array}{l}\text { Main Public Law Chapter 488: An act to } \\
\text { prevent opiate abuse }\end{array}$ & $\begin{array}{l}\text { "I think that whenever the government gets } \\
\text { involved in [medical] treatment, it is inappropriate." }\end{array}$ \\
\hline & $\begin{array}{l}\text { Criminalization of OUD and the } \\
\text { introduction of street heroin }\end{array}$ & $\begin{array}{l}\text { "You start with a bunch of OxyContin, you develop } \\
\text { the disease of addiction, and so you want to } \\
\text { continue with either opioid medication or heroin } \\
{[\ldots] \text {. As prescription medications started to be more }} \\
\text { expensive and harder to get, heroin was becoming } \\
\text { otherwise." }\end{array}$ \\
\hline \multirow[t]{2}{*}{ The problem of pain } & $\begin{array}{l}\text { OUD arising from both physical } \\
\text { and psychological pain. }\end{array}$ & $\begin{array}{l}\text { "There is no such difference as physical and } \\
\text { emotional pain [...]. Trauma causes emotional pain } \\
\text { and puts people at risk for opioid addiction. } \\
\text { Opioids [also] treat emotional pain." }\end{array}$ \\
\hline & $\begin{array}{l}\text { Vulnerability to OUD arising from influence } \\
\text { of social isolation, exclusion, and stigma }\end{array}$ & $\begin{array}{l}\text { "The opposition of addiction is not sobriety, but } \\
\text { social connection." }\end{array}$ \\
\hline
\end{tabular}


scribed by our informants, health care providers were unaware that the newer forms of opiates were no less addictive than those used previously. Encouraged by pharmaceutical companies' statements of safety and efficacy, physicians increasingly prescribed ER opiates. ${ }^{32}$ Similarly to our informants, other researchers have also reported that physicians treating pain as a fifth vital sign contributed to opioid over prescription patterns. ${ }^{32,33}$

The understanding of OUD as a chronic disease, as noted by our informants, has also been prevalent in the research, which documented that long-term opioid use leads to brain rewiring, mediated by persistent alterations to the function of reward-processing networks in the brain. ${ }^{34}$ The epigenetic changes result in further alterations to signaling cascades, cellular structure, and synaptic activities, leading to modifications critical to the formation and recall of long-term memory. ${ }^{34}$ These underlying physiological mechanisms contribute to the development of cravings, relapse, and addiction cycles, and demonstrate why it is difficult to discontinue the use of opioids. ${ }^{34}$ These results prompt some researchers to conclude that OUD must be managed similarly to other chronic diseases. ${ }^{34}$ A report from the National Center on Addiction and Substance Abuse also described OUD as a "chronic brain disease." 35

Similar to smaller states, in Maine community leaders became involved as OUD began to strain social networks, community resources, and criminal justice system. In response to demand for opiates, illegal heroin networks emerged in predominately white populations living in Maine's small metropolitan and rural areas. ${ }^{32}$ Social beliefs and attitudes regarding OUD further complicated the problem of OUD and its treatment. Informants in this study noted that "addiction as chronic disease" is a useful way to destigmatize it. They argue that to prevent deaths from OUD, laws such as Chapter 488 need to be coupled with a significant increase in the availability of treatment and recovery plans, overseen primarily by medical communities. Nevertheless, a focus on "addiction as a chronic brain disease" does not fully capture the problem of pain.

\section{The problem of pain: A socio-ecological model}

Findings from this study indicate that the problem of pain, leading to opioid use, is more complex than the simple story of treating physical pain with ER opiates, leading to tolerance, dependence, and eventual addiction. The complexity of OUD and addressing the current crisis is summarized in the socio-ecological model (SEM) (Figure 1). The SEM model illustrates how the opioid epidemic emerged from dynamic interactions among a constellation of factors, including misleading information from the

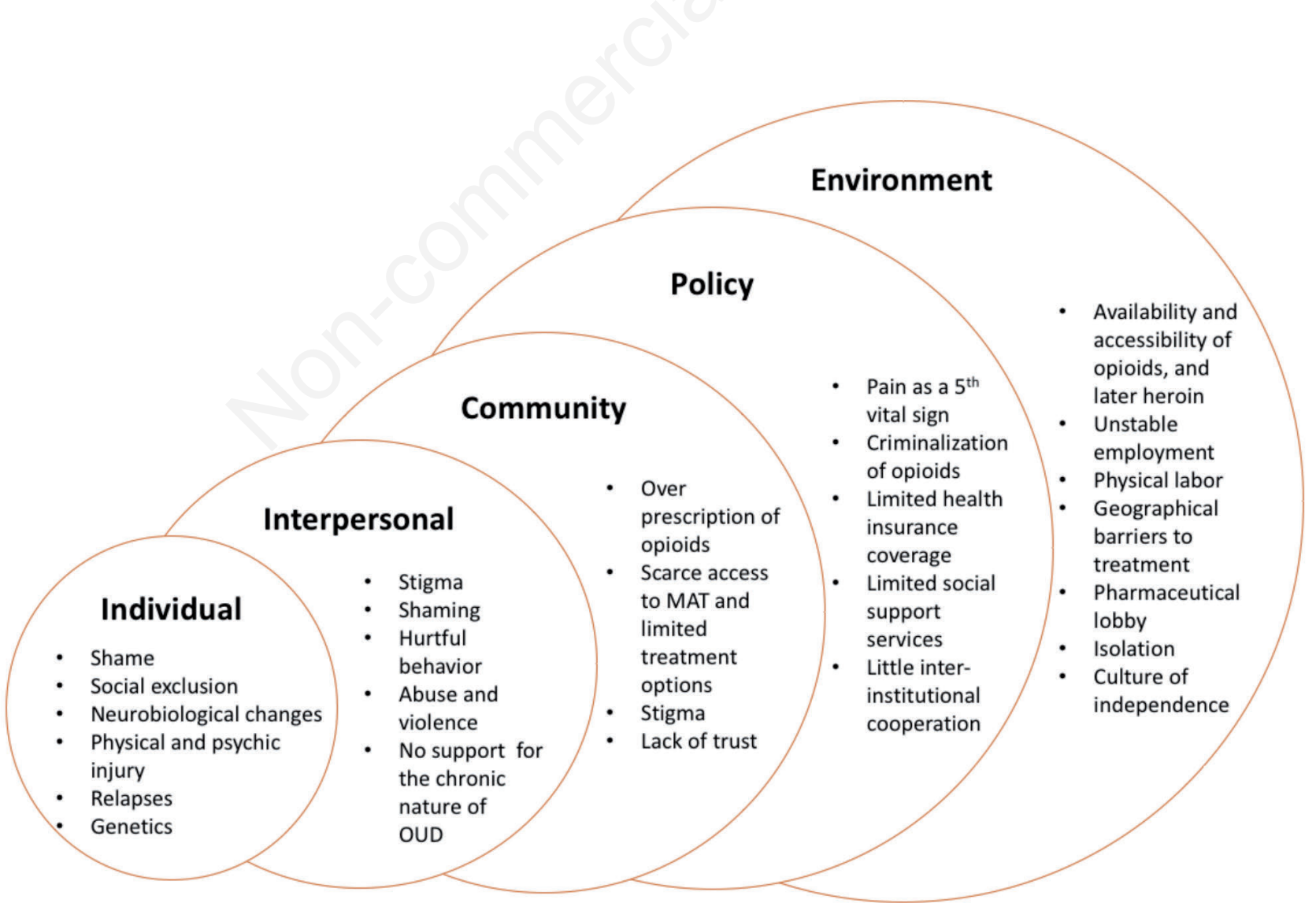

Figure 1. Socio-ecological model representing increasing vulnerability towards OUD in Maine, based on the factors reported by the interviewed health care professionals and community leaders. 
pharmaceutical lobby, over-prescription patterns, medical culture regarding pain treatment, and individuals' personal histories of pain, their sociodemographic characteristics, their communities' availability of supportive social structures, and local and regional cultural norms and policies regarding substance use. The United States' response to the problem of pain has deep historical and cultural roots that may have led to restrictive views of pain, narrowing treatment options and creating a fertile context for the opioid epidemic to emerge.

\section{The problem of pain: A historical, social perspective}

In modern American medical practice, pain has been considered difficult to measure objectively. ${ }^{36}$ Progress in modern medicine has developed through the rigor of "scientific objectivity" enabling medical researchers to develop diagnostic criteria for "real diseases." 36 The significance of "objectivity" was coupled with the establishment of mind-body dualism (in the $17^{\text {th }}$ century) and the belief in empirical or unbiased methods as the only legitimate paths to knowledge. ${ }^{37}$ The success of experimental sciences further reinforced the mind-body dualism as human beings were viewed predominantly as biological organisms (materialism) to be understood by examining their constituent parts (reductionism) using the principles of anatomy, physiology, biochemistry, and physics. ${ }^{31,36}$ As noted by several of our informants, attempts to objectify pain by use of a scale coupled with reductionists' approaches to treating pain have been counterproductive. Informants in this study believe that medical researchers and policymakers have overlooked the importance of individuals' socio-emotional context in the development of OUD.

\section{Pain as an integration of physical, psychological and socio- emotional factors}

The inclusion of psychological or "emotional injury" in the definition of pain would move the medical discourse beyond simplistic quantifiable measurements, such as the frequently used pain scale. ${ }^{38}$ Interestingly, neuroscience research has provided "objective" observations of pain through functional neuroimaging studies and prospective clinical studies demonstrating the differences between acute and chronic pain. ${ }^{38}$ The initial "acute" sensation of pain activates the sensory "pain matrix" region of the brain. ${ }^{38}$ However, chronic pain, impacts the underlying physiology of the brain related to dopamine circuits and brain regions involved in processing emotions, reward, and psychosocial events. ${ }^{38,39}$ Taking opioids for the first time makes the prevailing pain disappear and creates the powerful effect of disconnection from the stress and troubles of life, the "numbing" effects often described by health care providers in this study. Current medical practices, however, continue the anatomical focus and mindbody dualism split regarding the perception of pain. Yet, as demonstrated by this study, the process of understand- ing the complexity of pain is crucial in treating chronic pain and OUD.

\section{The role of social inclusion or rejection in $O U D$}

Though it can be initiated by replication of negative behavioral patterns within a peer group, OUD is also fueled by social isolation and rejection, reinforcing shame and stigma among those struggling with OUD. Neuroimaging research has revealed that the "unpleasantness" of physical pain activates the same regions of the brain that are stimulated during experiences of social rejection and self-reported social distress. ${ }^{40}$ These findings suggest that perception of pain and social distress may share neuronal circuits. ${ }^{40}$ Conversely, the presence of social support, which attenuates feelings of social distress, may alter perceptions of physical pain. Indeed, experimental work has shown that the presence of supportive others attenuates pain perception in both animals and humans. ${ }^{41}$ Therefore, presence of social support may be important in regulating perceptions of pain. ${ }^{41}$

As described by the study respondents, guilt and shame are also involved in shaping one's socio-environmental support structures. The feelings of guilt and shame, as part of stigmatization, can have various sources. Selfstigma is characterized as "negative feeling about self," while social stigma or structural stigma is related to restrictive rules, policies, and procedures. ${ }^{42}$ In healthcare setting, stigma is described as a socio-cultural process in which certain groups are devalued, rejected, and excluded on the basis of a socially discredited health conditions. ${ }^{42}$ The impact of labeling and stigmatization on service provision presents both the potential patients and allied health professionals with roadblocks to SUD treatment. Initiatives to establish new recovery centers are met with disagreement from local communities, arguing that they do not wish to have a "conglomeration of addicts hanging out in their neighborhood."

Characteristics of social connections in rural populations, such as geographical isolation and fixed social identities, can intensify patterns of stigmatization. Additionally, a paucity of social services in rural areas compound the problems of isolation. Accessibility to social support, followed by stability, structure, and re-connection are vital to help reverse "the tunnel like vision" and "chasing behind drugs," characteristic of drug-seeking behaviors. One of our respondents, Rosenberg, stated "The most effective recovery is to establish stability comprised of recognition, social support, and the possibility for a stable employment without mistrust and stigma".

The emphasis on developing new coping mechanisms to address personal and environmental challenges as well as to change past habits during recovery go beyond the treatment setting. Many of the people currently struggling with OUD lack skills to cope with the problems of everyday life. Therefore, expecting them to manage traumatic or burdensome events in a healthy way might be unrea- 
sonable. Further, if the propensity toward OUD begins in early childhood, through the environmental and social structures people grow up in, maybe the factors or sources fueling the current opioid epidemic should be searched for beyond medical practices and rather be explored within the context of deeper changes of societal or cultural characteristics of rural areas, which have taken place over the past decades.

\section{Conclusions}

OUD is a result of multifaceted behaviors which are strongly influenced by contextual factors and is difficult to encompass through any single lens (Figure 1). SUD results from dynamic interactions among an individual's biological and genetic predisposition, psychological constitution, and social environment and are best described through a biopsycho-social model. ${ }^{43}$ The way in which someone becomes introduced to substances - whether through self-medication or prescription medication- is yet another factor that informs the patterns of one's use. It is crucial to recognize that the formation of these behavioral patterns is further informed by social networks, their cohesion and identity, as well as by community practices around substance use and its legal and moral status, medical practice around OPMs, and social justice (Figure 1). Social policy can further define actions that affect the well-being of members of a society through shaping the distribution of and access to goods and resources (Figure 1). Those factors stress the interconnectedness between individual differences and situational and structural characteristics that form one's addictive behaviors. ${ }^{43}$

The current medical model of OUD continues to be reductionist. The assumption of a linear relationship between the brain and the drug implies that the complete removal of the drug or a genetic-based alteration in the brain should solve the problem of OUD. ${ }^{44}$ Historically, solutions addressing the problem of substance use through drug removal and criminalization often only increased the stigma and escalated the problem, as in the example of crack cocaine epidemic in earlier decades. ${ }^{45}$ Unfortunately, many models of OUD remain limited and therefore, are unable to equally represent interest of all involved, while some models can even put forth solutions mutually excluding interests of the involved groups. An example of such conflicting interests was described earlier as physicians argued for the use of MAT in jails.

Alternate conceptualizations of pain and SUD would have implications for several groups of people, including those with OUD, their families, researchers, medical providers, and policymakers. Any framework for the conceptualization of SUD must allow for a bottom-up development, integrated within the context of socio-ecological model of OUD development. ${ }^{43}$ Studying OUD as a broader cluster of factors would likely aid in broadening its comprehension and offering "multimodal approaches that include both individual and addiction-general treatments." ${ }^{\prime 46}$ Studies support the concept that patients with multiple comorbidities may benefit from the development and evaluation of integrated patient-centered interventions that target chronic pain, opioid dependence, and other psycho-social factors. ${ }^{47}$ Such an approach would require service providers to continually assess the impact of these factors on relapse and other treatment-related outcomes over the course of illness. ${ }^{46}$

Though promising, the implementation of such approaches requires an adequate recognition of what causes OUD in the first place and must provide people with an access to various treatment options which, in Maine and other rural areas, remains difficult either because of geographic limitations or sparseness of resources. ${ }^{19,48}$ Proposing that a client needs a certain amount of counseling for an extended period of time, different medications, or physical therapy to address chronic pain might be difficult in rural areas with limited providers and insufficient fiscal resources to ensure that services can be accessible to low income populations. ${ }^{18}$

OUD is a complex disease and as such, its understanding cannot be achieved through simplified and reductionist models. Focusing predominantly on OUD's physiology limits the financial and scientific potential to study and comprehend OUD and its implications in a broader, ecological context. By disregarding the complexity of OUD, scientific evidence leads to the creation and implementation of policies and treatment options which have a limited ability to help those struggling with the disease and its repercussions. With the current opioid overdose death rates, addressing such complex conditions through simplified models and promoting "one size fits all" solutions might have negative consequences and lead to a continuous increase in deaths from opioid overdoses. Most importantly, any theoretical framework or policy must remain faithful to and representative of lived human experience, or at the very least, it should allow space to meet the needs of the people whom it is intended to serve.

\section{Limitations}

There are multiple limitations in this study. Despite utilization of purposive sampling, we managed to interview only 58 per cent of the contacted providers and community leaders. Twenty-one of the interviewees were either medical providers or licensed counselors, leading to potential overrepresentation of their views in the study results. There were no interviews conducted with representatives of the pharmaceutical industry. Additional studies, with an in-depth focus on other stakeholders initially interviewed in this study (such as community leaders and criminal justice representatives), need to be conducted.

Limited funding and timeframe of this project, prevented us from accessing more remote communities in northern Maine, potentially limiting the generalizability 
of the results. Finally, Maine remains one of the least populated states with highest percentage of non-Hispanic whites in the US. ${ }^{49,50}$ Consequently, its inhabitants face different sets of challenges around their OUD and as such, their experiences may not be true, valid, or generalizable for inhabitants of other rural US states.

\section{References}

1. Public Law, Chapter 488. In: Maine So, ed. Vol SP0671 LD 1646. Augusta, Maine 2017

2. Gale JA, Hansen A, Elbaum Williamson M. Rural opioid prevention and treatment strategies: The experience in four states. 2017. Portland, ME: University of Southern Maine, Muskie School, Maine Rural Health Research Center; 2017, April.

3. Cicero TJ, Ellis MS, Surratt HL, Kurtz SP. Factors contributing to the rise of buprenorphine misuse: 2008-2013. Drug Alcohol Depend 2014;142:98-104.

4. Rudd RA, Seth P, David F, Scholl L. Increases in drug and opioid-involved overdose deaths-United States, 20102015. MMWR Morb Mortal Wkly Rep 2016; 65:1445-52.

5. Carroll JJ, Green TC, Noonan RK. Evidence-Based Strategies for Preventing Opioid Overdose: What's Working in the United States. National Center for Injury Prevention and Control, Centers for Disease Control and Prevention. 2018.

6. Warner M, Chen LH, Makuc DM, et al. Drug poisoning deaths in the United States, 1980-2008. NCHS data brief 2011:1-8.

7. Guy GP, Zhang K, Bohm MK, et al. Vital signs: Changes in opioid prescribing in the united states, 2006-2015. MMWR Morb Mortal Wkly Rep 2017; 66:697-04.

8. Musto DF. Opium, cocaine and marijuana in American history. Sci Am 1991;265:40-47.

9. Van Zee A. The promotion and marketing of oxycontin: Commercial triumph, public health tragedy. Am J Public Health 2009;99:221-7.

10. Rischitell DG, Karbowicz SH. Safety and efficacy of controlled-release oxycodone: A systematic literature review. J Am Coll Clin Pharm 2002:898-04.

11. Scholl L, Seth P, Kariisa M, et al. Drug and opioid-involved overdose deaths-United States, 2013-2017. MMWR Morb Mortal Wkly Rep 2019;67:1419.

12. Piper BJ, Lipovsky JW, Rodney MA, et al. Use and misuse of opioids in maine: results from pharmacists, the prescription monitoring, and the diversion alert programs. J Stud Alcohol Drugs 2016:556-65

13. Diomede T. SEOW: Special report: heroin, opioids, and other drugs in maine. state epidemiological outcomes workgroup. Augusta Maine: Department of Health and Human Serivces, the State of Maine. 2015 October.

14. National Institute on Drug Abuse. Maine: Opioid-involved deaths and related harms. Drug-Involved Overdose Deaths [website]. 2019; Accessed: April 20, 2020. Available from: https://www.drugabuse.gov/opioid-summaries-bystate/maine-opioid-involved-deaths-related-harms

15. Singh GK, Kim IE, Girmay M, et al. Opioid epidemic in the United States: Empirical trends, and a literature review of social determinants and epidemiological, pain management, and treatment patterns. Int J MCH AIDS 2019;8:89.

16. Sears JM, Edmonds AT, Fulton-Kehoe D. Tracking opioid prescribing metrics in washington state (2012-2017): Differences by County-level urban-rural and economic distress classifications. J Rural Health 2020;36:152-66.

17. Rigg KK, Monnat SM, Chavez MN. Opioid-related mortality in rural America: Geographic heterogeneity and intervention strategies. Int J Drug Policy 2018;57:119-29.

18. Thomas N, van de Ven K, Mulrooney KJ. The impact of rurality on opioid-related harms: A systematic review of qualitative research. Int J Drug Policy 2019:102607.

19. Lister JJ, Weaver A, Ellis JD, et al. A systematic review of rural-specific barriers to medication treatment for opioid use disorder in the United States. Am J Drug Alcohol Abuse 2019:1-16.

20. Joudrey PJ, Edelman EJ, Wang EA. Drive times to opioid treatment programs in urban and rural counties in 5 US states. JAMA 2019;322:1310-2.

21. Logan DE, Lavoie AM, Zwick WR, et al. Integrating addiction medicine into rural primary care: Strategies and initial outcomes. J Consult Clin Psychol 2019;87:952-61.

22. Jenkins RA, Hagan $H$. What is a rural opioid risk and policy environment?. Int J Drug Policy. 2019:102606.

23. Spradley JP. Participant observation. Waveland Press; 2016. In: Creswell JW, Creswell JD. Research design: Qualitative, quantitative, and mixed methods approaches. Thousand Oaks, CA: Sage publications; 2017.

24. Creswell JW, Plano Clark VL. Designing and conducting mixed method research. 2nd. Sage; Thousand Oaks, CA: 2011.

25. Chapman A, Hadfield M, Chapman C. Qualitative research in healthcare: An introduction to grounded theory using thematic analysis. J R Coll Physicians Edinb 2015;45:201-05.

26. Woods P, Gapp R, King MA. Generating or developing grounded theory: Methods to understand health and illness. Int J Clin Pharm 2016;38:663-70.

27. Miles MB. New methods for qualitative data collection and analysis: Vignettes and pre $\square$ structured cases. Int J Qual Stud Educ 1990;3:37-51.

28. Tong A, Sainsbury P, Craig J. Consolidated criteria for reporting qualitative research (COREQ): a 32-item checklist for interviews and focus groups. Int J Qual Health Care 2007; 19:349-57.

29. Clark B, Lane PS, Trent L, Cropsey KL. Methadone maintenance treatment may improve completion rates and delay opioid relapse for opioid dependent individuals under community corrections supervision. Addict Behav 2014:1736-40.

30. Caruth C. Unclaimed experience: Trauma and the possibility of history. Yale Fr Stud 1991:181-92.

31. Mehta N. Mindbody dualism: A critique from a health perspective. Mens Sana Monogr 2009:202-09.

32. Cicero TJ, Ellis MS. The prescription opioid epidemic: A review of qualitative studies on the progression from initial use to abuse. Dialogues Clin Neuro 2017;19:259.

33. Ballantyne JC, Sullivan MD. Intensity of chronic pain-the wrong metric? N Engl J Med 2015;373:2098-99.

34. Browne CJ, Godino A, Salery M, Nestler EJ. Epigenetic mechanisms of opioid addiction. Biol Psychiatry 2020; 87:22-33.

35. Altman DE, Fineberg H, Gold M, et al. Addiction medicine: Closing the gap between science and practice. Columbia University, The National Center on Addiction and Substance Abuse, New York. 2012.

36. Goldberg DS. The Bioethics of Pain Management: Beyond Opioids. New York City, NY: Routledge; 2014. 
37. Crane T, Patterson S. History of the Mind-Body Problem. New York City, NY: Routledge; 2001.

38. Hashmi J, Huang L, Baria A, et al. Shape shifting pain: Chronification of back pain shifts brain representation from nociceptive to emotional circuits. Brain 2013:2761-68.

39. Taylor AM, Schweinhardt P, Cahill C. Mesolimbic dopamine signaling in acute and chronic pain: implications for motivation, analgesia, and addiction. Pain 2016:1194-98.

40. Eisenberger NI, Jarcho JM, Lieberman MD, Naliboff BD. An experimental study of shared sensitivity to physical pain and social rejection. Pain 2006;126:132-8.

41. Hsu DT, Meyers KK, Love TM, et al. Response of the $\mu$ opioid system to social rejection and acceptance. Mol Psychiatry 2013:1211-17.

42. Livingston JD, Milne T, Fang ML, Amari E. The effectivness of interventions for reducing stigma related to substnce use disorders: a systematic review. Addict 2011:39-50.

43. Griffiths M. A 'component' model of addiction within a biopsychosocial framework. J Subst Use 2005:191-7.

44. Hart C. Viewing Addiciton as a brain disease promotes social injustice. Nat Hum Behav 2017;1.
45. Tyler ET, Brockmann B. Returning home: Incarceration, reentry, stigma and the perpetuation of racial and socioeconomic health inequity. J Law Med Ethics 2017;45:545-57.

46. Shaffer HJ, LaPlante DA, LaBrie RA, et al. Toward a syndrome model of addiciton: Multiple expressions, common etiology. Harv Rev Psychiatry 2004:367-74.

47. Barry DT, Cutter CJ, Beitel M, et al. Psychiatric disorders among patients seeking treatment for co-occuring chronic pain and opioid use diosrder. J Clin Psychiatry 2016:1-7.

48. Rosenblatt RA, Andrilla CHA, Catlin M, Larson EH. Geographic and specialty distribution of US physicians trained to treat opioid use disorder. Ann Fam Med 2015;13:23-26.

49. Maine State GOvernment. Maine Population Outlook 20162026. 2018. Augusta, Maine. Accessed: March 172020. Available from: https://www.maine.gov/dafs/economist/sites/ maine.gov.dafs.economist/files/inline-files/Maine\%20Population\%20Outlook\%20to\%202026.pdf

50. United State Census Bureau. QuickFacts Maine, USA. 2018. Accessed on: March 17 2020. Retrived from: https:// www.census.gov/quickfacts/ME 